\title{
Determination of the migration effect and molecular docking of verteporfin in different subtypes of breast cancer cells
}

\author{
CHANGRAN WEI ${ }^{1}$ and XIANGQI LI ${ }^{1,2}$ \\ ${ }^{1}$ Department of The First Clinical Medical School, Shandong University of Traditional Chinese Medicine, Jinan, \\ Shandong 250355; ${ }^{2}$ Department of Breast Surgery, The Second Affiliated Hospital of Shandong First Medical University, \\ Tai'an, Shandong 271000, P.R. China
}

Received February 2, 2020; Accepted July 28, 2020

DOI: $10.3892 / \mathrm{mmr} .2020 .11482$

\begin{abstract}
Breast cancer is one of the most aggressive malignant tumors in women. According to the expression differences of estrogen receptor, progesterone receptor, human epidermal growth factor receptor-2 (HER-2) and cell proliferation antigen $\mathrm{Ki}-67$, breast cancer can be divided into four molecular subtypes: Luminal A, Luminal B, HER-2 overexpression and Basal-like. Yes-associated protein (YAP), a downstream effector of the Hippo pathway, is overexpressed in human cancers and is associated with proliferation, apoptosis, migration, invasion and resistance to chemotherapy drugs in breast cancer cells. Verteporfin (VP) is used as a photosensitizer in the treatment of neovascular macular degeneration. VP is also identified as an inhibitor of YAP/TEA domain transcription factor (TEAD) interaction in the absence of light activation. However, detailed structural information about VP and YAP interactions is relatively scarce and VP research targeting YAP in different molecular subtypes of breast cancer cells is also rare. The aims of the present study were to structurally describe the VP binding site in the YAP crystal structure and to verify the non-photoreactive VP effect targeting YAP on the migration of different molecular subtypes of breast cancer cells. The crystal structure of VP and YAP was calculated by AutoDock 4.2 and the result was illustrated using PyMOL. The non-photoactivated VP effect on the migration of Luminal A MCF-7, Luminal B BT-474 and triple-negative breast cancer BT-549 breast cancer cells was evaluated by wound healing and Transwell migration experiments. Results from molecular docking experiments demonstrated that VP could interact through hydrogen bonds and hydrophobic interactions with important YAP residues
\end{abstract}

Correspondence to: Professor Xiangqi Li, Department of Breast Surgery, The Second Affiliated Hospital of Shandong First Medical University, 366 Taishan Road, Tai'an, Shandong 271000, P.R. China E-mail: drlixqi@hotmail.com

Key words: verteporfin, yes-associated protein, inhibitor, yes-associated protein/transcriptional enhanced associate domain complex, migration, non-photoinduced therapy involved in TEADs binding (Gln82, Val84, Met86 and Arg89). Migration experiments revealed that the non-photoinduced VP could inhibit the migration of different molecular subtypes of breast cancer cells. The results of the present study indicated that VP may be a novel repositioned drug for breast cancer treatment in the future.

\section{Introduction}

The Hippo signaling pathway serves an important role in the occurrence and progression of breast cancer (1). As a terminal effector of a central kinase cascade of the Hippo signaling pathway, dephosphorylation and nuclear localization of yes-associated protein (YAP) regulates the activity of many YAP downstream compounds, including connective tissue growth factor (CTGF), cysteine-rich angiogenic inducer (CYR61), AXL receptor tyrosine kinase (AXL) and integrin, when combined with the DNA-binding transcription factors of TEA domain transcription factor (TEAD) (2). CYR61, CTGF and integrin serve an important role in cell adhesion, migration, proliferation and angiogenesis (3). Overexpression of YAP in breast cancer promotes the occurrence and development of breast cancer, while YAP knockdown can reduce cell proliferation, migration and increase the radiotherapy sensitivity of triple-negative breast cancer (TNBC) 231 cells $(4,5)$. Activated YAP is a key regulator of tumor cell proliferation, apoptosis, migration, invasion and chemotherapy resistance, leading to cell malignant transformation (6). Taken together, these studies indicate that YAP may be a potential drug target for breast cancer.

Verteporfin (VP) was originally designed as a photosensitizer for macular degeneration (7). VP can produce singlet oxygen and eliminate abnormal hyperplastic blood vessels under the activation of a $693 \mathrm{~nm}$ laser (8). In recent years, VP has lost its importance owing to the limited efficacy of its original indications (9). However, VP also serves a key role in the absence of light, VP can inhibit YAP-TEAD binding without photoactivation and markedly prevent liver hepatomegaly/tumorigenesis induced by YAP overexpression (10). YAP is closely associated with the occurrence and development of breast, colon, lung and liver cancer in addition to mesothelioma, as it is the core effector of the Hippo signaling pathway (11). Therefore, targeting YAP is may be 
an attractive therapeutic strategy. VP was rapidly repositioned as a YAP inhibitor for several types of cancer, including liver, esophageal, lung and pancreatic cancer(12).

Non-photoactivated VP can disrupt the interaction and transcriptional activity of YAP/TEAD and thus inhibit YAP-mediated tumor proliferation, induce tumor cell apoptosis and restore sensitivity to chemotherapy drugs (13). However, although VP has been identified as a YAP/TEAD inhibitor, a detailed structural analysis of YAP and VP interactions has yet to be presented. Therefore, the present study analyzed the possible binding sites of VP and YAP by molecular simulation. In addition, it evaluated the VP effect on the migration of three breast cancer cell subtypes and on the expression of YAP downstream cell migration-related genes.

\section{Materials and methods}

Compound preparation. VP (MedChemExpress) was dissolved in DMSO as a stock solution at a concentration of $10 \mathrm{mM}$, stored at $-20^{\circ} \mathrm{C}$ and protected from light.

Breast cancer cell culture. Luminal A MCF-7, Luminal B BT-474 and TNBC BT-549 cells (14) were purchased from Shanghai Zhongqiao Xinzhou Biotechnology Co., Ltd. The cells were cultured with RPMI-1640 medium (HyClone; Cytiva) supplemented with $10 \%$ fetal bovine serum (Gibco; Thermo Fisher Scientific, Inc.). Cells were incubated at $37^{\circ} \mathrm{C}$ in a humidified atmosphere of $95 \%$ air and $5 \% \mathrm{CO}_{2}$. The cells were protected from light at all times.

Molecular docking studies. All molecular docking studies were performed using AutoDock 4.2.6 software package (15). The crystal structure of YAP (PDB code $3 \mathrm{KYS}$ ) was retrieved from the RCSB Protein Data Bank (https://www.rcsb. org/) (16). The structure was prepared by adding hydrogen atoms, removing waters and adding Gasteiger charge. The three-dimensional structure of VP was retrieved from Drugbank 5.1.5 (https://www.drugbank.ca/) and was prepared by adding hydrogen atoms and Gasteiger charge. The protonation states of both receptor protein and VP were determined at pH 7 using Propka 3.1 (17). Then the ligand was docked into the binding sites of YAP with default parameters and 20 docking poses were exported for further visual analysis. The docking score of each pose was calculated with AutoDock 4.2. The binding free energy was of best-scored pose calculated using the Molecular Mechanics/Poisson-Boltzmann Surface Area (MM-PBSA) method (13). Fig. 1 presents the best-scored binding pose of VP with YAP docking result, which was constructed with PyMOL 2.4.0 (https://pymol.org/2/).

Wound healing assay. Three parallel lines were drawn in advance on the bottom of a 24-well plate. MCF-7, BT-474 and BT-549 cells were placed in the plates at $1.2 \times 10^{5}$ cells per well. After the cells reached $85-90 \%$ confluence, a $10 \mu$ l pipette tip was used to draw a light, straight line. The floating cells in the wells were carefully washed off with PBS buffer and images captured under the light microscope at the $0 \mathrm{~h}$ time point. The blank control group cells were cultured in fresh medium without FBS and the treatment group was treated with $8 \mu \mathrm{M}$ VP. After $24 \mathrm{~h}$ of culture, the cells were washed with PBS buffer three times and images captured under the microscope. Image-Pro Plus 6.0 software (Media Cybernetics, Inc.) was used to quantify the migration distance of cells before and after scratches and to calculate the wound healing distance.

Transwell assay. For these experiments, the cells were cultured at a density of $3 \times 10^{5}$ in FBS-free medium. In the control group, $10 \%$ FBS medium was dripped along the side wall of the lower Transwell chamber. In the treatment group, 10\% FBS medium containing $8 \mu \mathrm{M}$ VP was added to the lower Transwell chamber. The $3 \times 10^{4}$ cell suspensions were carefully added to the upper Transwell chamber and then cultured at $37^{\circ} \mathrm{C}$ in the incubator for $24 \mathrm{~h}$. The medium was then removed, and the cells were washed twice with PBS and fixed for 20 min with $4 \%$ paraformaldehyde at room temperature. The sample were stained with DAPI for $15 \mathrm{~min}$ in the dark and images captured under a fluorescence microscope (magnification, x100). A total of five visual fields were randomly selected and the results were quantified by Image Pro Plus 6.0 software (Media Cybernetics, Inc.).

Statistical analysis. Data are presented as the mean \pm standard deviation. Comparisons between VP-treated and -untreated control groups were made by unpaired t-test. $\mathrm{P}<0.05$ was considered to indicate a statistically significant difference.

\section{Results}

Molecular docking analysis. The crystal structure of YAP (PDB code 3KYS) was used as docking receptor for VP in docking molecular experiments and was prepared by adding hydrogen atoms, removing waters and adding Gasteiger charge. The best-scored pose of VP was selected as the possible bind conformation for the docking analysis (Fig. 1) and the docking score was -9.73. VP can form a hydrogen bond with the side chain of Gln82 and the distance was $2.1 \AA$ (O...H-N-Gln82). In addition, VP could have a hydrogen bond with the main chain of Val84 at the distance of $1.8 \AA$ (O...H-N-Val84). VP can also form a hydrogen bond with the main chain of Met86 at the distance of $2.0 \AA$ (O...H-N- Met86). VP can establish two hydrogen bonds with the positively charged Arg89 side chain at distances of $1.8 \AA(\mathrm{O}$...H-N-Arg89) and $1.7 \AA$ (O...H-N-Arg89), respectively. In addition, VP established hydrophobic interactions with the surrounding residues Leu68, Ala71, Val72, Pro75, Val80, Pro81, Val84, Pro85 and Met86. These interactions tightly bound to YAP and inhibited the interaction of YAP and TEAD. The binding free energy was further investigate using MM-PBSA and the binding free energy was $-32.16 \mathrm{kcal} / \mathrm{mol}$, which was favorable for the binding of VP. This result suggested that VP was a good inhibitor for the YAP/TEAD complex.

$V P$ effect on the migration of different breast cancer cell subtypes. The expression of YAP target proteins in MCF-7, BT-474 and BT-549 cells was evaluated. Western blotting demonstrated that $8 \mu \mathrm{M}$ VP treatment could notably downregulate the protein expression levels of YAP, TEAD, and CYR61. The expression of CTGF were differential in three subtypes of breast cancer and no CTGF expression was detected in MCF-7 cells (Fig. 2). 


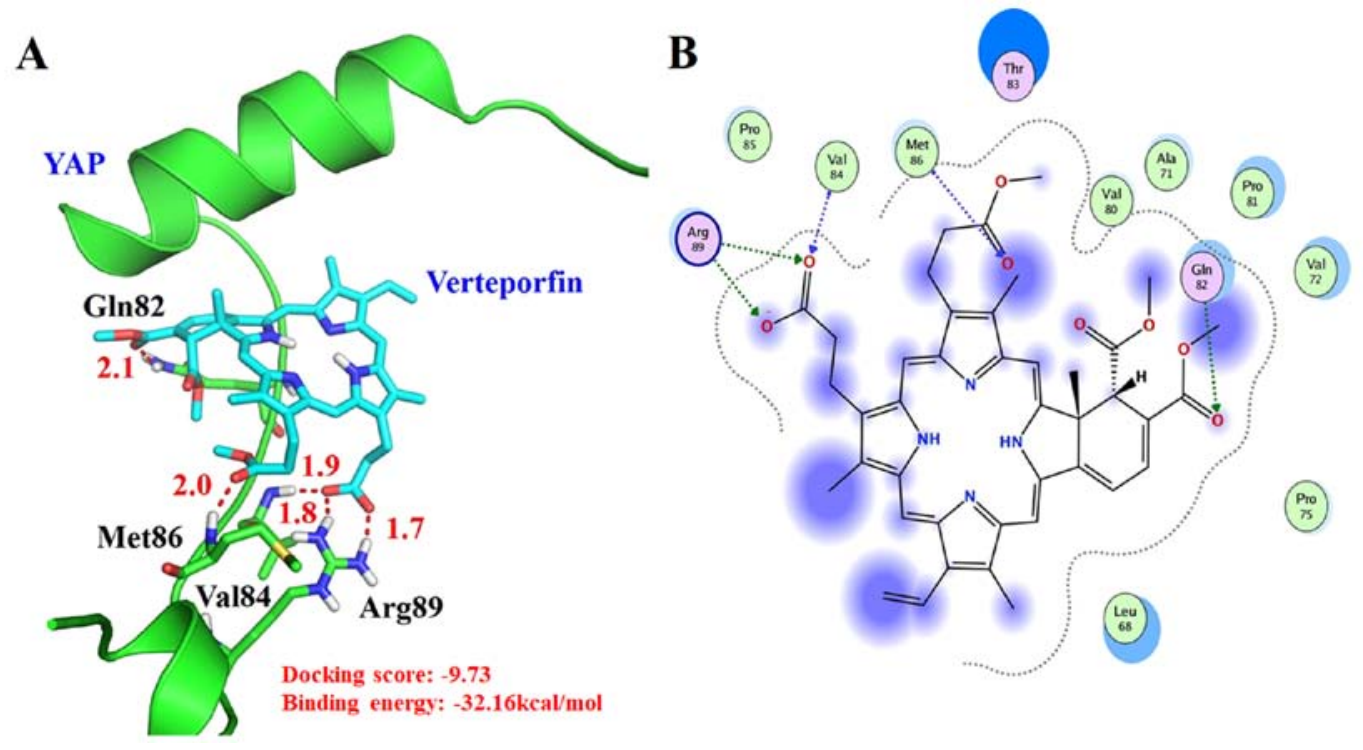

Figure 1. Molecular docking analysis (A) Docking results of verteporfin interaction with protein surface and (B) surrounding residues of YAP (PDB code 3KYS). VP is shown in green stick model and key residues of YAP were shown in cyan stick models. The nitrogen, oxygen and sulfur atoms were shown in blue, red and yellow, respectively. The hydrogen bonds are represented by red dashed lines. YAP, yes-associated protein.
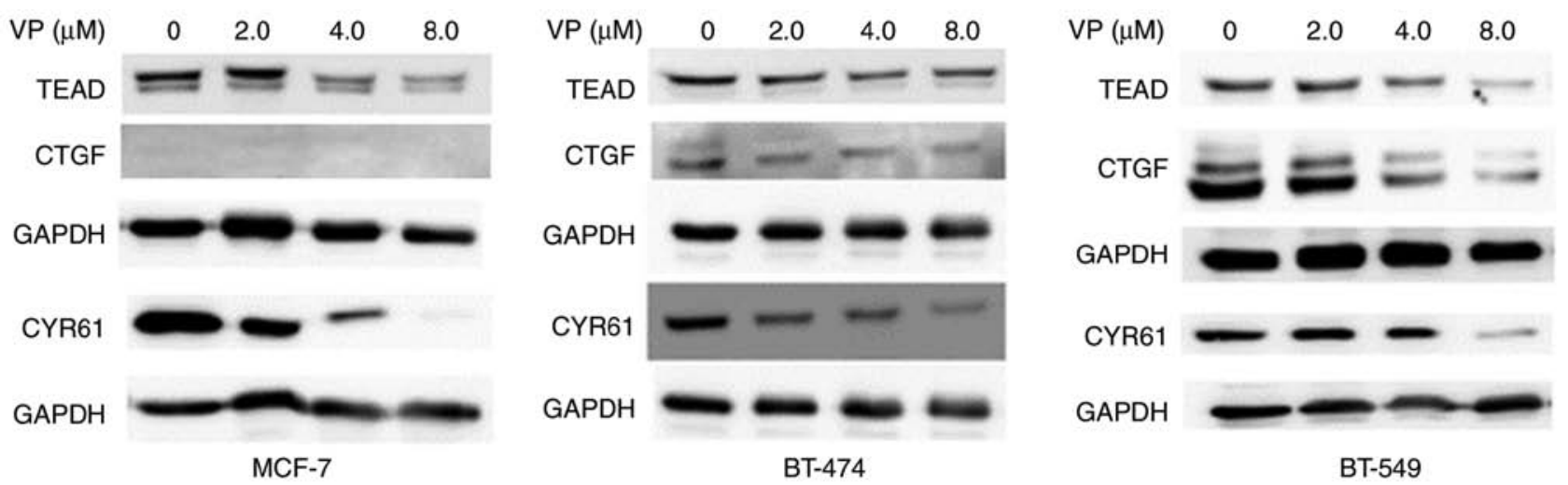

Figure 2. Effects of VP on the protein expression levels of downstream YAP targets. CTGF, connective tissue growth factor; CYR61, cysteine-rich angiogenic inducer; TEAD, TEA domain transcription factor; YAP, yes-associated protein; VP, verteporfin.

Transwell migration assays revealed that $8 \mu \mathrm{M}$ VP could inhibit the number of migratory cells of Luminal A MCF-7, Luminal B BT-474 and TNBC BT-549 cells (P<0.01; Fig. 3). Wound healing assay results demonstrated that $8 \mu \mathrm{M}$ VP decreased the migratory ability of the three different breast cancer cell subtypes (Fig. 4).

\section{Discussion}

The core components of the Hippo signaling pathway are involved in the regulation of proliferation, migration, invasion and chemoresistance of breast cancer cells (11). YAP is the main effector of the Hippo signaling pathway (1). The core kinases Mst1/2 of the Hippo signaling pathway and the activation complex formed by the interaction of their regulatory protein, protein salvador homolog 1 , can directly phosphorylate large tumor suppressor homolog (LATS)1 and LATS2, which interact with Mob kinase activator 1 (Mob1). The activity of LATS1/LATS2 and Mob1 can be inhibited by YAP phosphorylation, which is degraded by proteasome or ubiquitination in the cytoplasm (4). By contrast, when the upstream kinase signal of the Hippo pathway is inhibited, the unphosphorylated Yap is transferred to the nucleus (12). Owing to the lack of a DNA-binding domain, YAP must be combined with DNA-binding transcription factors to serve the role of transcription coactivators (18). YAP overexpression can induce epithelial-mesenchymal transition in the MCF-10A breast cell line and overexpression of YAP promotes the formation and growth of tumor in breast cancer cells in a mouse xenograft tumor model (19). The activation of YAP in tumor cells can ultimately promote the occurrence of breast cancer bone metastasis (20). These data therefore suggest that YAP is closely associated with the occurrence and development of breast cancer. Since it was identified as an inhibitor of the YAP/TEAD complex, several studies have reported the therapeutic potential of VP in different types of cancer (21-23). However, detailed structural information on the interaction between VP and YAP remains lacking. The N-terminal YAP region contains a TEAD-binding domain and 14-3-3-binding domain site (HXRXXS motif-containing Ser127 residue) (24). 

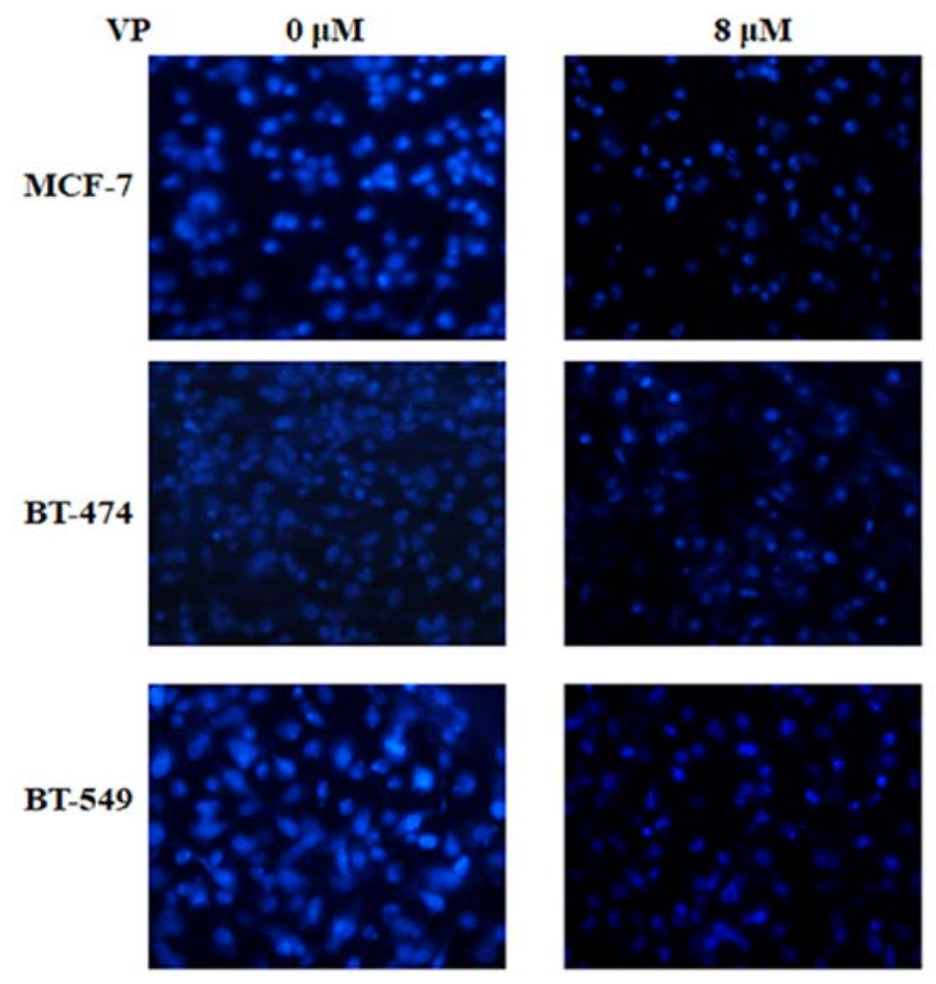
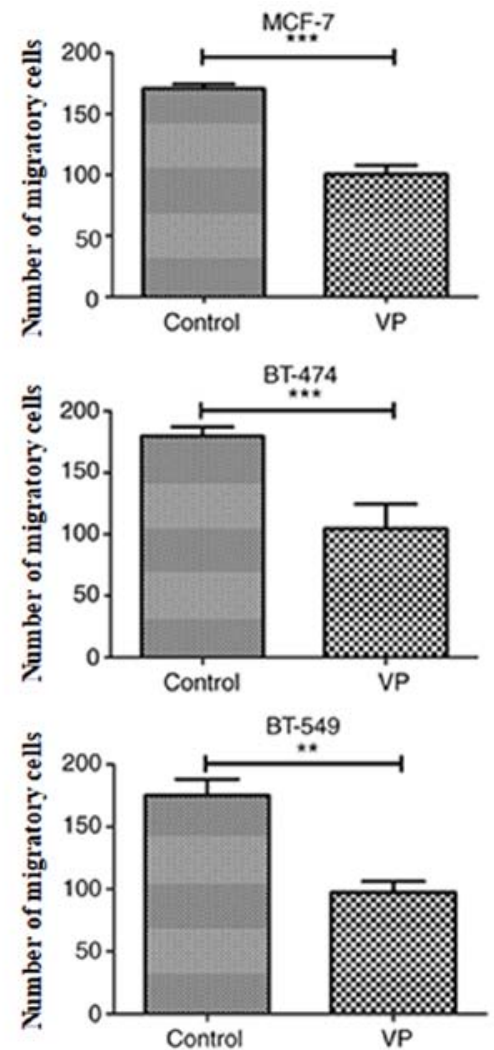

Figure 3. VP affects the migratory ability of three breast cancer cell subtypes: MCF-7, BT-474 and BT-549. ${ }^{* *} \mathrm{P}<0.01,{ }^{* * *} \mathrm{P}<0.001$ vs. control group. VP, verteporfin.

YAP binding to 14-3-3 proteins depends on Ser127 phosphorylation, which also serves a key role in determining the cytoplasmic localization and inactivation of YAP $(25,26)$. YAP-TEAD interaction is important for the YAP-mediated transcription activation and the disruption of this interaction is being considered as a strategy in cancer therapy (27). Liu-Chittenden et al found that VP selectively binds to YAP and abrogates its interaction with TEAD (10). In recent years, some crystal structures have been presented, including YAP (residues 47-85)-TEAD4 (residues 210-427) complex from Mus musculus (PDB code 3JUA), YAP (residues 50-171)-TEAD1 (residues 209-426) from Homo sapiens (PDB code 4RE1) and YAP (residues 60-100)-TEAD4 (residues 217-434) complexes from Homo sapiens (PDB code 3KYS) (28). Structure analysis has shown that the 61-100 region of human YAP serves an essential role in TEAD-binding domain, it is observed that the hydrophobic interaction of the YAP $\Omega$-ring formed by Met86, Arg87, Leu91, Phe95 and Phe96 residues causes the Arg89 and Ser94 side chains to form hydrogen bonds with Glu255, Asp264 and Tyr421 residues of TEAD1 (28-30).

YAP and TEAD proteins are the best molecular target candidates to regulate the Hippo pathway, as the formation and activation of the YAP/TEAD complex is the last step of the Hippo-YAP pathway. VP is a compound that directly destroys the formation of YAP/TEAD complexes and inhibits the most essential part of the Hippo pathway (10). The molecular docking analysis of the present study found that VP could form hydrogen bonds and establish hydrophobic interactions with residues from the YAP $\Omega$-ring that directly interacted with TEAD1 (Met86 and Arg89). Our molecular docking results demonstrated that VP could also interact with several surrounding residues (Leu68, Ala71, Val72, Pro75, Val80, Pro81, Gln82, Val84 and Pro85), particularly by hydrophobic interactions, highlighting that it occupies the TEAD binding site and inhibits the YAP-TEAD complex formation.

YAP is highly expressed in many solid tumors and its carcinogenic function is mediated by its nuclear localization and interaction with TEAD transcription factors $(31,32)$. By interfering with YAP/TEAD, non-photoactivated VP can inhibit the proliferation of esophageal (33), lung (34) and pancreatic cancer (21), in addition to malignant mesothelioma cells (35) and bladder cancer cells (22), inducing cell apoptosis. VP can significantly inhibit the transcriptional activity of TEAD in MDA-MB-231 cells, reduce the protein expression level of YAP target genes AXL and CTGF, inhibit the migration of paclitaxel resistant cells and induce apoptosis in vitro. VP reduces tumor volume and Ki67 expression in paclitaxel-resistant mice and reverses the resistance of TNBC tumor cells to paclitaxel therapy in vivo (23). The overexpression of YAP promotes resistance to chemotherapy, radiotherapy and targeted treatment of various types of cancer (13). Non-photoactivated VP can also increase the sensitivity of HER-2 positive breast cancer cells to lapatinib (36) and increase the sensitivity of TNBC MDA-MB-231 cells (37), urothelial cells (38), esophageal cancer cells (30) and colon cancer cells (39) to chemotherapy drugs. Therefore, non-photoactivated VP may provide a new choice for drug-targeted YAP intervention in breast cancer.

Abnormal expression of CTGF and Cyr61 proteins is associated with the progression of breast cancer, prostate cancer and malignant melanoma $(3,40,41)$. The present study found that CTGF were expressed in Luminal B BT-474 and TNBC BT-549 breast cancer cells and no CTGF expression 

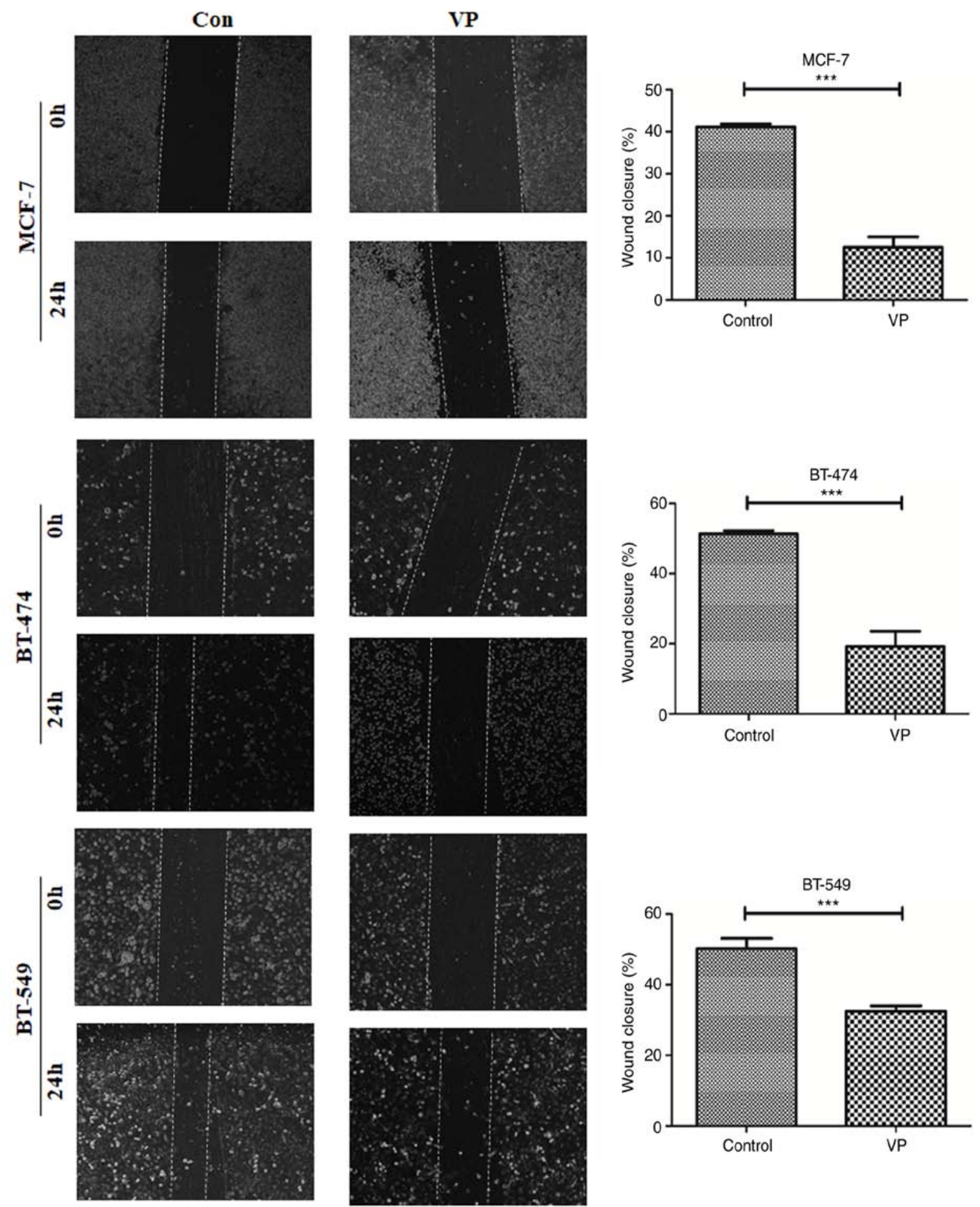

Figure 4. VP effect on the wound healing ability of three breast can cell subtypes: MCF-7, BT-474 and BT-549. Magnification, $x 100 .{ }^{* * *}$ P $<0.001$ vs. control group. VP, verteporfin.

was detected in Luminal A MCF-7 cells, which was in accord with previous data (42). CTGF specific antibody can reduce the bone metastasis incidence in a mouse model of breast cancer (43). The present study found that non-photoactivated VP can inhibit the migration of three breast cancer cell lines in vitro by downregulating the expression of YAP/TEAD downstream target genes CYR61, which are closely associated with cell migration and invasion.
In conclusion, VP is an FDA-approved photosensitizer for the treatment of age-related macular degeneration and can inhibit the occurrence of tumor by destroying the formation of YAP/TEAD complex in its non-photoactivated form (14). In the present study, the molecular interface between VP and YAP was simulated and the binding site between VP and YAP was confirmed by molecular docking experiment. VP, a non-photoactive inhibitor of Hippo-YAP signaling, could inhibit the 
migration of three molecular subtypes of breast cancer cells by targeting YAP. The potential role of VP in cancer progression, including cell proliferation and apoptosis, without light activation need to be further studied and evaluated in experimental animals and human breast cancer treatment. The results of the present study provided a new approach for targeting the Hippo pathway effector YAP to treat breast cancer.

\section{Acknowledgements}

Not applicable.

\section{Funding}

The present study was supported by The National Natural Science Foundation of China (grant no. 81473687), the Shandong Provincial Natural Science Foundation, China (grant nos. ZR2009CM039 and ZR2013HM038), the High Level Project Cultivation Program of Shandong First Medical University, China (grant no. 2018GCC14) and the Academic promotion of Shandong First Medical University, China (grant no. 2019QL017).

\section{Availability of data and materials}

The datasets used and/or analyzed during the present study are available from the corresponding author on reasonable request.

\section{Authors' contributions}

CW acquired and analyzed the data, and drafted and revised the manuscript. XL conceptualized the study, acquired and analyzed the data, and drafted and revised the manuscript. Both authors read and approved the final manuscript.

\section{Ethics approval and consent to participate}

Not applicable.

\section{Patient consent for publication}

Not applicable.

\section{Competing interests}

The authors declare that they have no competing interests.

\section{References}

1. Fu V, Plouffe SW and Guan KL: The Hippo pathway in organ development, homeostasis, and regeneration. Curr Opin Cell Biol 49: 99-107, 2017.

2. Holden JK and Cunningham CN: Targeting the hippo pathway and cancer through the TEAD family of transcription factors Cancers (Basel) 10: 81, 2018.

3. Xie D, Nakachi K, Wang H, Elashoff R and Koeffler HP: Elevated levels of connective tissue growth factor, WISP-1, and CYR61 in primary breast cancers associated with more advanced features. Cancer Res 61: 8917-8923, 2001.

4. Luo XL, Yan Q, Tao DD, Hu JB, Li ZM, Li XL and Gong JP: Effects of MST1 on cell proliferation and apoptosis of human carcinoma cell line MCF-7. Tumor 28: 852-854, 2008.
5. Chen Q, Zhang N, Gray RS, Li H, Ewald AJ, Zahnow CA and Pan D: A temporal requirement for Hippo signalling in mammary gland differentiation, growth and tumorigenesis. Genes Dev 28: 432-437, 2014

6. Heidary Arash E, Shiban A, Song S and Attisano L: MARK4 inhibits Hippo signaling to promote proliferation and migration of breast cancer cells. EMBO Rep 18: 420-436, 2017.

7. Scott LJ and Goa KL: Verteporfin. Drugs Aging 16: 139-146; discussion 147-148, 2000.

8. Richter AM, Waterfield E, Jain AK, Allison B, Sternberg ED, Dolphin D and Levy JG: Photosensitising potency of structural analogues of benzoporphyrin derivative (BPD) in a mouse tumour model. Br J Cancer 63: 87-93, 1991.

9. Ziemssen $\mathrm{F}$ and Heimann $\mathrm{H}$ : Evaluation of verteporfin pharmakokinetics-redefining the need of photosensitizers in ophthalmology. Expert Opin Drug Metab Toxicol 8: 1023-1041, 2012.

10. Liu-Chittenden Y,Huang B, Shim JS, Chen Q, Lee SJ, Anders RA, Liu JO and Pan D: Genetic and pharmacological disruption of the TEAD-YAP complex suppresses the oncogenic activity of YAP. Genes Dev 26: 1300-1305, 2012.

11. Plouffe SW, Hong AW and Guan KL: Disease implications of the Hippo/YAP pathway. Trends Mol Med 21: 212-222, 2015.

12. Santucci M, Vignudelli T, Ferrari S, Mor M, Scalvini L, Bolognesi ML, Uliassi E and Costi MP: The hippo pathway and YAP/TAZ-TEAD protein-protein interaction as targets for regenerative medicine and cancer treatment. J Med Chem 58: 4857-4873, 2015

13. Gibault F, Corvaisier M, Bailly F, Huet G, Melnyk P and Cotelle P: Non-photoinduced biological properties of verteporfin. Curr Med Chem 23: 1171-1184, 2016.

14. Neve RM, Chin K, Fridlyand J, Yeh J, Baehner FL, Fevr T, Clark L, Bayani N, Coppe JP, Tong F, et al: A collection of breast cancer cell lines for the study of functionally distinct cancer subtypes. Cancer Cell 10: 515-527, 2006.

15. Miller BR III, McGee TD Jr, Swails JM, Homeyer N, Gohlke H and Roitberg AE: MMPBSA.py: An efficient program for end-state free energy calculations. J Chem Theory Comput 8: 3314-3321, 2012.

16. Berman HM, Battistuz T, Bhat TN, Bluhm WF, Bourne PE, Burkhardt K, Feng Z, Gilliland GL, Iype L, Jain S, et al: The protein data bank. Acta Crystallogr D Biol Crystallogr 58 (Pt 6 No 1): 899-907, 2002.

17. Rostkowski M, Olsson MH, Søndergaard CR and Jensen JH: Graphical analysis of $\mathrm{pH}$-dependent properties of proteins predicted using PROPKA. BMC Struct Biol 11: 6, 2011.

18. Yu FX, Zhao B and Guan KL: Hippo pathway in organ size control, tissue homeostasis, and cancer. Cell 163: 811-828, 2015.

19. Overholtzer M, Zhang J, Smolen GA, Muir B, Li W, Sgroi DC, Deng CX, Brugge JS and Haber DA: Transforming properties of YAP, a candidate oncogene on the chromosome 11q22 amplicon. Proc Natl Acad Sci USA 103: 12405-12410, 2006.

20. Li C, Wang S, Xing Z, Lin A, Liang K, Song J, Hu Q, Yao J, Chen Z, Park PK, et al: A ROR1-HER3-lncRNA signalling axis modulates the Hippo-YAP pathway to regulate bone metastasis. Nat Cell Biol 19: 106-119, 2017.

21. Wei H, Wang F, Wang Y, Li T, Xiu P, Zhong J, Sun X and Li J: Verteporfin suppresses cell survival, angiogenesis and vasculogenic mimicry of pancreatic ductal adenocarcinoma via disrupting the YAP-TEAD complex. Cancer Sci 108: 478-487, 2017.

22. Dong L, Lin F, Wu W, Liu Y and Huang W: Verteporfin inhibits YAP-induced bladder cancer cell growth and invasion via Hippo signaling pathway. Int J Med Sci 15: 645-652, 2018.

23. Shi G, Wang H, Han H, Gan J and Wang H: Verteporfin enhances the sensitivity of LOVO/TAX cells to taxol via YAP inhibition. Exp Ther Med 16: 2751-2755, 2018.

24. Harvey KF, Zhang X and Thomas DM: The Hippo pathway and human cancer. Nat Rev Cancer 13: 246-257, 2013.

25. Moroishi T, Hansen CG and Guan KL: The emerging roles of YAP and TAZ in cancer. Nat Rev Cancer 15: 73-79, 2015.

26. Mesrouze Y, Meyerhofer M, Bokhovchuk F, Fontana P, Zimmermann C, Martin T, Delaunay C, Izaac A, Kallen J, Schmelzle T, et al: Effect of the acylation of TEAD4 on its interaction with co-activators YAP and TAZ. Protein Sci 26: 2399-2409, 2017.

27. Pobbati AV, Han X, Hung AW, Weiguang S, Huda N, Chen GY, Kang C, Chia CS, Luo X, Hong W, et al: Targeting the central pocket in human transcription factor TEAD as a potential cancer therapeutic strategy. Structure 23: 2076-2086, 2015. 
28. Gibault F, Sturbaut M, Bailly F, Melnyk P and Cotelle P. Targeting transcriptional enhanced associate domains (TEADs). J Med Chem 61: 5057-5072, 2018.

29. Li Y, Liu S, Ng EY, Li R, Poulsen A, Hill J, Pobbati AV, Hung AW, Hong W, Keller TH and Kang C: Structural and ligand binding analysis of the YAP-binding domain of transcription factor TEAD4. Biochem J 475: 2043-2055, 2018.

30. Tian W, Yu J, Tomchick DR, Pan D and Luo X: Structural and functional analysis of the YAP-binding domain of human TEAD2. Proc Natl Acad Sci USA 107: 7293-7298, 2010.

31. Lin KC, Park HW and Guan KL: Regulation of the hippo pathway transcription factor TEAD. Trends Biochem Sci 42: 862-872, 2017.

32. Vlug EJ, Van De Ven RA, Vermeulen JF, Bult P, van Diest PJ and Derksen PW: Nuclear localization of the transcriptional coactivator YAP is associated with invasive lobular breast cancer. Cell Oncol (Dordr) 36: 375-384, 2013.

33. Song S, Honjo S, Jin J, Chang SS, Scott AW, Chen Q, Kalhor N, Correa AM, Hofstetter WL, Albarracin CT, et al: The Hippo coactivator YAP1 mediates EGFR overexpression and confers chemoresistance in esophageal cancer. Clin Cancer Res 21: 2580-2590, 2015.

34. Kim J, McMillan E, Kim HS, Venkateswaran N, Makkar G, Rodriguez-Canales J, Villalobos P, Neggers JE, Mendiratta S, Wei S, et al: XPO1-dependent nuclear export is a druggable vulnerability in KRAS-mutant lung cancer. Nature 538: 114-117, 2016.

35. Tranchant R, Quetel L, Tallet A, Meiller C, Renier A, de Koning L, de Reynies A, Le Pimpec-Barthes F, Zucman-Rossi J, Jaurand MC and Jean D: Co-occurring mutations of tumor suppressor genes, LATS2 and NF2, in malignant pleural mesothelioma. Clin Cancer Res 23: 3191-3202, 2016.

36. Lin C, Pelissier FA, Zhang H, Lakins J, Weaver VM, Park C and LaBarge MA: Microenvironment rigidity modulates responses to the HER2 receptor tyrosine kinase inhibitor lapatinib via YAP and TAZ transcription factors. Mol Biol Cell 26: 3946-3953, 2015.
37. Li Y, Wang S, Wei X, Zhang S, Song Z, Chen X and Zhang J: Role of inhibitor of yes-associated protein 1 in triple-negative breast cancer with taxol-based chemoresistance. Cancer Sci 110: 561-567, 2019.

38. Ciamporcero E, Shen H, Ramakrishnan S, Yu Ku S, Chintala S, Shen L, Adelaiye R, Miles KM, Ullio C, Pizzimenti S, et al: YAP activation protects urothelial cell carcinoma from treatment-induced DNA damage. Oncogene 35: 1541-1543, 2016.

39. Li W, Cao Y, Xu J, Wang Y, Li W, Wang Q, Hu Z, Hao Y, Hu L, Sun Y, et al: YAP transcriptionally regulates COX-2 expression and GCCSysm-4 (G-4), a dual YAP/COX-2 inhibitor, overcomes drug resistance in colorectal cancer. J Exp Clin Cancer Res 36: 144, 2017.

40. Yang F, Tuxhorn JA, Ressler SJ, McAlhany SJ, Dang TD and Rowley DR: Stromal expression of connective tissue growth factor promotes angiogenesis and prostate cancer tumorigenesis. Cancer Res 65: 8887-8895, 2005.

41. Hutchenreuther J, Vincent KM, Carter DE, Postovit LM and Leask A: CCN2 expression by tumor stroma is required for melanoma metastasis. J Invest Dermatol 135: 2805-2813, 2015.

42. Li MH, Sanchez T, Pappalardo A, Lynch KR, Hla T and Ferrer F: Induction of antiproliferative connective tissue growth factor expression in Wilms' tumor cells by sphingosine-1-phosphate receptor 2. Mol Cancer Res 6: 1649-1656, 2008.

43. Shimo T, Kubota S, Yoshioka N, Ibaragi S, Isowa S, Eguchi T, Sasaki A and Takigawa M: Pathogenic role of connective tissue growth factor (CTGF/CCN2) in osteolytic metastasis of breast cancer. J Bone Miner Res 21: 1045-1059, 2006.

This work is licensed under a Creative Commons Attribution-NonCommercial-NoDerivatives 4.0 International (CC BY-NC-ND 4.0) License. 